\title{
The Human 230-kD Bullous Pemphigoid Antigen Gene (BPAG1) Exon-Intron Organization and Identification of Regulatory Tissue Specific Elements in the Promoter Region
}

Katsuto Tamai, Daisuke Sawamura, Haeyoung Choi Do, Yoshiko Tamai, Kehua Li, and Jouni Uitto Departments of Dermatology, and Biochemistry and Molecular Biology, Jefferson Medical College, and the Section of Molecular Dermatology, Jefferson Institute of Molecular Medicine, Thomas Jefferson University, Philadelphia, Pennsylvania 19107

\begin{abstract}
The 230-kD bullous pemphigoid antigen (BPAG1), a hemidesmosomal protein, is encoded by a gene at the human chromosomal locus 6p11-12. We have elucidated the exon-intron organization of the entire human BPAG1 gene, including $\sim 2.6 \mathrm{~kb}$ of 5 -flanking DNA. Seven overlapping genomic clones, spanning $\sim 20 \mathrm{~kb}$, contained the entire $\sim 9 \mathrm{~kb}$ coding sequence of BPAG1 and consisted of 22 separate exons, which varied from 78 to 2,810 bp in size. The $5^{\prime}$ flanking region of DNA, upstream from the ATG initiation codon for translation, was found to contain several putative transcriptional response elements. Most interestingly, two motifs potentially conferring keratinocyte specific expression to the gene were detected. The presence of such elements was suggested by $\sim 20$-fold higher expression of a promoter/chloramphenicol acetyl transferase (CAT) construct in normal human epidermal keratinocytes that express the endogenous gene, as compared to several nonexpressing cell types. Transient transfections with 5'-deletion clones of the promoter / reporter gene (CAT) constructs identified a region containing a putative tissue specific element, KRE2, which also conferred tissue specificity to the expression of the truncated promoter downstream from this element, however, a mutated derivative of KRE2 was not functional. Detailed knowledge of the structure and regulation of the BPAG1 gene will aid in further elucidation of diseases affecting the cutaneous basement membrane zone. (J. Clin. Invest. 1993. 92:814822.) Key words: hemidesmosomal proteins • keratinocyte specific expression - blistering skin diseases - epidermolysis bullosa
\end{abstract}

\section{Introduction}

Bullous pemphigoid (BP $)^{1}$ is a cutaneous blistering disease characterized by the presence of circulating IgG autoantibodies in the patients' sera $(1,2)$. Two autoantigens recognized by these antibodies have been characterized. The major autoanti-

Address reprint requests to Jouni Uitto, M.D., Ph.D., Thomas Jefferson University, Department of Dermatology, 233 South 10th Street, Room 450, Philadelphia, PA 19107.

Received for publication 28 August 1992 and in revised form 1 March 1993.

1. Abbreviations used in this paper: BPAG1, 230-kD bullous pemphigoid antigen; BP, bullous pemphigoid; CAT, chloramphenicol acetyl transferase; EB, epidermolysis bullosa; KRE2, keratinocyte responsive element 2; KTF, keratinocyte transcription factor; NHF, human skin fibroblasts; NHK, normal human keratinocytes.

J. Clin. Invest.

(C) The American Society for Clinical Investigation, Inc. 0021-9738/93/08/0814/09 \$2.00

Volume 92, August 1993, 814-822 gen is a $230-\mathrm{kD}$ noncollagenous protein, bullous pemphigoid antigen-1 (BPAG1 ), recognized by most patients' sera, while a minor autoantigen is a $180-\mathrm{kD}$ collagenous protein (BPAG2) recognized by the sera of some patients with $B P$, and also by the sera of patients with herpes gestationis, a pregnancy-associated blistering disease $(3,4)$. Immunoelectron microscopic studies have indicated that these two autoantigens are associated with hemidesmosomes, attachment complexes at the cutaneous basement membrane zone between the basal keratinocytes and the underlying lamina lucida $(5,6)$. It has been suggested that the intermolecular interactions of the BPAGs with intracellular intermediate filaments and with cell surface macromolecules, such as $\alpha 6 \beta 4$ integrins together with anchoring filament proteins and type VII collagen, may secure the stable association of the epidermis to the underlying basement membrane (7). Thus, BPAGs may play a critical role in the overall stability of the cutaneous basement membrane zone, although direct evidence in support of this suggestion is lacking.

The 230-kD BP antigen has been recently cloned (8-11), and the corresponding human gene, BPAG1, has been localized to the short arm of chromosome 6 at locus 6 p11-12 (12). Elucidation of the primary sequences of BPAG1, as deduced from the composite full-length cDNA, revealed the presence of distinct domain structures (11). In the central portion of the molecule, the presence of three subdomains characterized by heptad repeats has predicted an $\alpha$-helical coiled-coil structure. This central portion of the protein is flanked by two globular domains. In the carboxyl-terminal end of the molecule, eight repeat segments of 38-amino acid residues, each with a high degree of homology with consensus sequences in desmoplakin I, a component of desmosomal cytoplasmic plaque, were detected $(10,11,13)$. The amino-terminal end has features characteristic of a globular domain. Thus, we have proposed a molecular structure for BPAG1, consisting of a dimer with central $\alpha$-helical coiled-coil region, flanked by $\mathrm{NH}_{2}$ - and $\mathrm{COOH}$-terminal globular domains (11).

In addition to the acquired autoimmune diseases, BP antigens have been suggested to be candidate genes in some forms of epidermolysis bullosa (EB), a group of genodermatoses characterized by extreme fragility of the skin and the mucous membranes (7). In particular, abnormalities in the hemidesmosomes have been demonstrated by ultrastructural analysis in the junctional forms of EB. Since the expression of the 230kD BP antigen is limited to epidermal keratinocytes, not detectable in dermal fibroblasts (9), the characterization of the underlying mutations and elucidation of the mechanisms of the tissue specific expression may necessitate direct analysis of DNA at the genomic level. Toward this aim, we have undertaken characterization of the human BPAG1 gene. In this study we report the complete exon-intron organization of the BPAGl gene. We have also characterized the 5'-flanking DNA and identified regulatory segments, as well as sequences potentially conferring tissue specificity of expression. 


\section{Methods}

Isolation and characterization of BPAGI genomic clones. A human lung fibroblast genomic DNA library in $\lambda$ FIX II phage vector (Stratagene, La Jolla, CA) was screened with four different segments of the composite cDNA corresponding to BPAG1 sequences (Fig. $1 \mathrm{~A}$ ). These cDNAs have been previously shown to correspond to 8,930 bp of nucleotide sequences in BPAG1 mRNA, which contains a coding segment for 2,649 amino acids (11). The recombinant genomic clones were purified, and the inserts were characterized by restriction enzyme digestions and by Southern analysis with synthetic oligonucleotides based on exon sequences derived from the cDNA (14). The endonuclease digestion products were fractionated by electrophoresis on $1 \%$ agarose gels, and their sizes were estimated by comparison to standard DNA markers (New England Biolabs, Inc., Beverly, MA). Appropriate DNA fragments were isolated by gel electrophoresis and subcloned into the Bluescript vector (Stratagene) for sequencing. Some genomic clones were subjected to digestion with a $5^{\prime} \rightarrow 3^{\prime}$ exonuclease (T7 gene 6; United States Biochemical Corp., Cleveland, $\mathrm{OH}$ ) (15), and the resulting single stranded DNA was directly used as a template for sequencing. The nucleotide sequencing was performed using dideoxynucleotide chain termination method (16), utilizing universal and reverse primers, as well as synthetic oligonucleotide primers that were synthesized on the basis of sequence information in the BPAG1 cDNA. The nucleotide sequences were translated to putative peptide sequences and sequence alignments as well as search for homology with consensus sequences were performed by the pc/Gene program (IntelliGenetics, Inc./Betagen, Mountain View, CA).

The sizes of the introns in the genomic DNA were either determined by direct nucleotide sequencing or estimated by generating PCR products using synthetic oligonucleotide primers in the flanking exons, as identified in the full-length cDNA (11). The sizes of the PCR products were estimated from agarose gels in comparison to standard DNA markers.

5 ' primer extension of the $m R N A$ sequences. Primer extensions of the mRNA sequences at the $5^{\prime}$ end of the BPAG1 transcript were performed with 0.2 pmol of ${ }^{32}$ P-labeled oligonucleotide PE1, 5'TTTTATTCCTGAAACGATGTTCTT-3', using T4 polynucleotide kinase and $30 \mu \mathrm{g}$ of total RNA as template (14). The reaction was extended by the addition of $20 \mathrm{U}$ of reverse transcriptase (Promega Biotec, Madison, WI), and the mixture was incubated for $60 \mathrm{~min}$ at $42^{\circ} \mathrm{C}$. The radioactive product was directly fractionated on $10 \%$ polyacrylamide gel containing $8 \mathrm{M}$ urea. Radioactively labeled standard DNA size markers were run in adjacent lanes. Also, a positive control, as included in the commercial kit (Promega), and a negative control that excluded the reverse transcriptase, were run in parallel. The primer extension products were visualized by autoradiography on $\mathrm{x}$-ray films (X-Omat; Eastman-Kodak, Rochester, NY).

Construction of BPAGI promoter/CAT reporter gene plasmids. One of the genomic clones $(\lambda 1)$ was shown to contain the 5 ' end of the coding region and flanking DNA sequences (see Results). To develop BPAG1 promoter/reporter gene (CAT) chimeric constructs, a doublestranded DNA extending from position -1 to $-1,907$ (counting upstream from the translation initiation site) was first generated by PCR amplification. The primers used for PCR were $27 \mathrm{bp}$ oligomers, and they contained an Xhol site in the 5 ' oligomer and a HindIII site in the 3 ' oligomer, which enabled direct cloning of the PCR product into a pBSOCAT, a promoterless construct containing the entire CAT gene, small $\mathbf{t}$ intron, and the polyadenylation signal in plasmid Bluescript (pBSKS) (17); this construct is referred to as pBP1.9CAT. Four additional constructs with the 3 ' end of the promoter being fixed at -1 , and the 5 ' end position varying from $-1,427$ to -519 (constructs pBP1.4, pBP0.9, pBP0.7 and pBP0.5CAT) were developed by a similar PCR strategy. All PCR generated clones were sequenced, and no PCR errors were detected in comparison to the original genomic sequence used as template. To extend the promoter region toward the 5 ' end, an additional clone, pBP2.6CAT, was generated by isolation of a Xbal-AccI fragment from the genomic $\lambda 1$ clone. This fragment was ligated to the
5 ' end of the pBP1.4CAT clone after it was truncated by digestion with the AccI endonuclease. Thus, the pBP2.6CAT construct extended from -1 to $-2,637$. Furthermore, an additional 5 ' deletion clone was generated by KpnI digestion of pBP2.6CAT to yield the construct pBP2.5CAT with the 5 ' end at $-2,509$.

During the promoter analysis, a nucleotide sequence, which potentially conferred tissue specificity to the expression of the BPAG1 gene in epidermal keratinocytes, was discovered in the position $-[1,887$ to 1,898 ] (see Results). The functionality of this sequence, which we referred to as KRE2, was tested by cloning it in front of pBP1.4CAT to create the pKRE2/BP1.4CAT construct. Furthermore, a similar construct in which the KRE2 sequence was mutated by substituting two cytosine residues by adenosines ( see text) was developed; this construct was designated as pMKRE2/BP1.4CAT.

The promoter/CAT constructs, as well as pBSOCAT (a negative control), $5 \mu \mathrm{g} / 28 \mathrm{~cm}^{2}$ plate, were used in transient transfections of normal human epidermal keratinocytes $\left(3 \times 10^{5} /\right.$ plate $)$ cultured in serum-free KGM medium, as described previously $(18,19)$. In some experiments, human oral epidermoid carcinoma cells (KB), HeLa cells, normal human skin fibroblasts (NHF), mouse NIH-3T3 transformed fibroblasts and human transformed amniotic epithelial (WISH) cells were transfected in parallel. The transfections were performed with a commercial kit (Transfectam; Promega) (20). In all transfections, the BPAG1 constructs were cotransfected with a SV $2 \beta \mathrm{Gal}$ construct, which was used as an internal control of transfection efficiency. Following a 24-h incubation, CAT activity as an indicator of the promoter activity was determined by incubation with $\left[{ }^{14} \mathrm{C}\right]-$ chloramphenicol, as previously described $(17,21)$. $\beta$-Galactosidase activity was determined in the same samples (14), and the CAT activity values were corrected for $\beta$-galactosidase activity to allow direct comparison of the BPAG1 promoter activity in different cell cultures transfected in parallel.

Northern analyses. To examine the expression of the BPAGl gene at the mRNA steady-state level, total RNA was isolated by a single-step extraction procedure (22) from the same type of cultured cells that were used for transient transfections (see above). Total RNA ( $30 \mu \mathrm{g}$ per lane) was fractionated in $1.0 \%$ agarose gels, and the prehybridizations and hybridizations were performed with a human BPAG1 cDNA, as described previously (9). The same filters were also hybridized with a human GAPDH cDNA, to verify even loading of the RNA to each lane (9).

\section{Results and Discussion}

Isolation and characterization of BPAGl genomic clones. Screening of a human genomic DNA $\lambda$ phage library with four different BPAG1 cDNAs (pcBPA-4, 6, 7 and 10; see Fig. $1 A$ [11]) identified 15 positive clones, and 7 of them $(\lambda 1-7)$ were characterized in detail by restriction enzyme digestions, Southern analysis, and dideoxynucleotide sequencing. Digestion of the insert DNA with a variety of restriction enzymes, followed by Southern blot analyses, allowed their precise alignment (Fig. $1 B$ ). The inserts varied from $\sim 9$ to $14 \mathrm{~kb}$ in size, and the composite of the overlapping genomic DNA corresponding to the coding region of BPAG1 spanned $\sim 20 \mathrm{~kb}$ (Fig. $1 \mathrm{~B}$ ). Since the size of the BPAG1 mRNA is $\sim 9 \mathrm{~kb}$, the exon-intron ratio of the gene is $\sim 0.82$, indicating a relatively high coding density. Digestions with $\mathrm{XbaI}$ and SacI restriction enzymes allowed isolation of subclones for nucleotide sequencing (Fig. 1 $B$ ). Dideoxynucleotide sequencing with oligomer primers synthesized on the basis of sequences previously identified in the cDNA or complementary to new sequences detected in the introns allowed identification of the exon-intron borders and subsequent determination of the exon sizes (Figs. $1 C$ and 2). Specifically, the cloned genomic DNA was used as a template 
A.

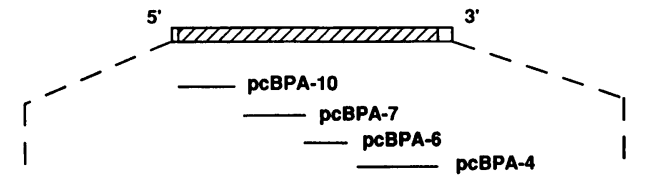

B.
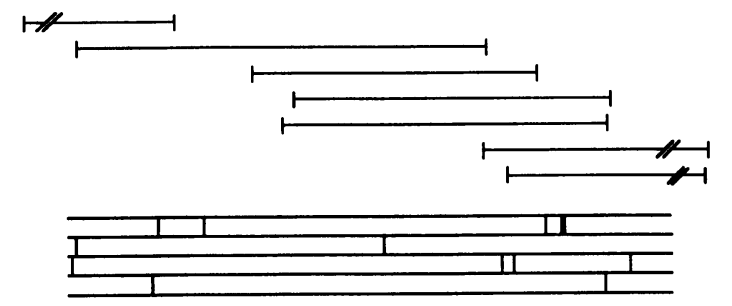

c.

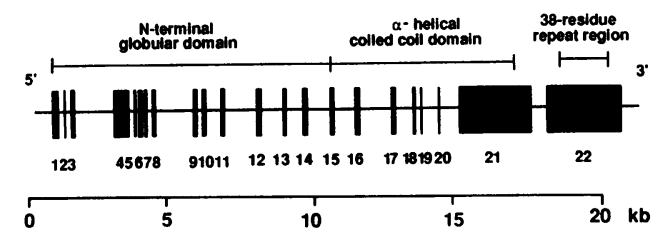

Figure 1. Isolation of genomic DNA clones corresponding to the human BPAGl gene. $(A)$ Four separate cDNAs (pcBPA) corresponding to different segments of the full-length mRNA were used to screen a genomic DNA $\lambda$ FIX library. $(B)$ Seven distinct genomic clones $(\lambda 1-7)$, varying from 9 to $14 \mathrm{~kb}$ in size, were isolated. Restriction enzyme digestions with XbaI, SacI, Accl, and DraIII endonucleases allowed precise alignment of the clones. The segment corresponding to the coding region of BPAG1 mRNA spanned $\sim 20 \mathrm{~kb}$ of genomic sequences. $(C)$ The entire coding region of the BPAG1 consisted of 22 exons numbered starting from the 5 ' end, drawn to scale. The individual exons are depicted by vertical bars, and the introns are shown by horizontal lines. The gene segments corresponding to the $\mathrm{NH}_{2}$-terminal globular domain, $\alpha$-helical coiled coil domain, and a 38-amino acid residue repeat region are indicated.

to prime sequencing reactions, and the sequences were compared with the established cDNA sequences (11). New oligomer primers were synthesized to prime a reverse sequencing reaction so that all exon-intron borders were sequenced in both directions. Sequencing of all seven phage clones indicated that the 7,948 bp open reading frame in the composite full-length BPAG1 cDNA consisted of 22 separate exons (Fig. $1 C$ ).

Exon-intron organization of the human BPAGl gene. Elucidation of the individual exon-intron junctions indicated the presence of a consensus motif ag/X at the acceptor splice site, which was preceded by a pyrimidine tract, and the donor site displayed a consensus sequence X/gt (22) (Fig. 2). The codons at the splice site did not demonstrate a characteristic pattern, and the codon for the first amino acid could be split in any three different combinations. This absence of a casettelike arrangement of the intron-exon junctions mitigates against alternative splicing of the pre-mRNA (23), and in fact, no evidence for alternative splicing has been observed by comparison of overlapping cDNAs isolated from different cDNA libraries (8-11).

The sizes of the individual exons varied from 78 to $2810 \mathrm{bp}$ (Fig. 2). Interestingly, the $3^{\prime}$ end of the gene corresponding to the 38-amino acid residue repeat region was encoded by a single large exon, no. 22 (Fig. $1 C$ ). The 7 exons in the region coding for the three $\alpha$-helical coiled-coil subdomains varied from 84 to $2,730 \mathrm{bp}$ in size. However, two of the subdomains were encoded by a single exon (no. 21) without interruptions, while the most upstream of the three subdomains was encoded by separate exons (nos. 15-17; Fig. $1 C$ ). The region corresponding to the amino terminal globular domain consisted of 15 exons varying from 78 to $591 \mathrm{bp}$ in size. It should be noted that the exon at the junction between the $\mathrm{NH}_{2}$-terminal globular domain and the $\alpha$-helical coiled-coil region (exon no. 15) was a fusion exon shared by both domains. These observations indicate that the different protein domains of the $230-\mathrm{kD}$ BP antigen are encoded by gene segments depicting distinct intron-exon organizations. These arrangements may relate to the stability of the gene with the potential for rearrangements conceivably resulting in heritable diseases. In particular, the segments containing intron sequences are more likely to undergo rearrangements potentially mediated by $A l u$ and other repetitive elements within the intron sequences $(24,25)$.

When the genomic exon sequences derived from the $\lambda F I X$ II library were compared with the published BPAG1 cDNA

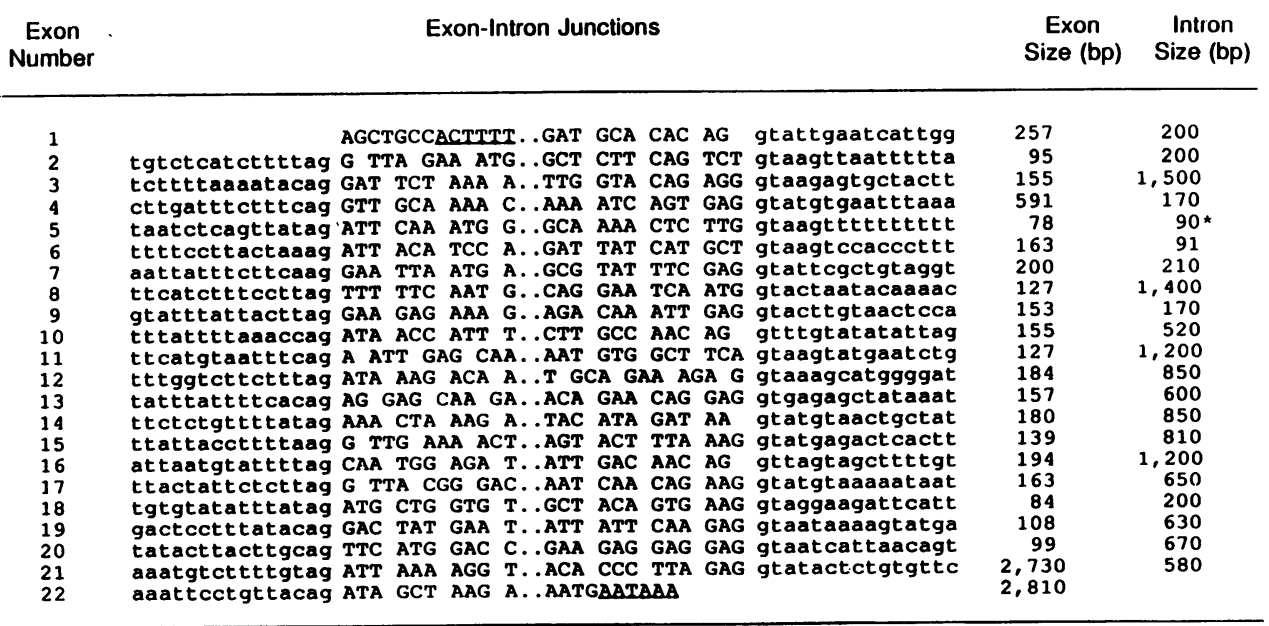

Figure 2. Boundaries at the exon-intron junctions, and the sizes of the exons and introns of human BPAG1 gene. The exon numbers correspond to those shown in Fig. $1 C$. Nucleotide sequences in the introns are indicated by lowercase letters, and those in exons by capital letters. Note the presence of a consensus cap sequence, ACTTTT (underlined), at the 5 ' of the exon 1 . However, RT-PCR and primer extensions identified the 5 ' end of the mRNA to reside $7 \mathrm{bp}$ upstream of this site (see text and Figs. 3 and 4 ). Also note the presence of a polyadenylation consensus sequence, AATAAA, at the $3^{\prime}$ end of the exon 22 (underlined). The exon sizes were determined by direct nucleotide sequencing of the corresponding cDNAs. The intron sizes were determined either by nucleotide sequencing $\left({ }^{*}\right)$ or estimated from the sizes of PCR products synthesized by using oligonucleotide primers in the flanking exons. Note that some of the exons begin with a complete codon for an amino acid, while others demonstrate the presence of split codons. 
sequences, six variant nucleotides were detected. These substitutions resulted in change of four amino acids, three of the substitutions being conservative. These changes were a Gly $\rightarrow$ Val substitution at the amino acid position 851 in reference 10 , $\mathrm{Thr} \rightarrow$ Asp at 1,045, Glu $\rightarrow$ His at 1,624, and Gly $\rightarrow$ Arg at 1,943 . The reasons for the differences between cDNA and genomic sequences are not clear, but these substitutions may reflect allelic polymorphism of the BPAG1 gene. In this context, it is of interest to note that two restriction fragment length polymorphisms (RFLPs) have been previously identified within this gene $(9,26)$. One of these, a MspI RFLP, has been used for genetic linkage analysis to exclude the BPAG1 gene as a candidate gene in a large kindred with dominant EB simplex (26). Nevertheless, these RFLPs can be used to examine other families with different forms of EB for cosegregation of a BPAG1 allele with the clinical phenotype (7).

Elucidation of the 5' and $3^{\prime}$ untranslated segments of the BPAGI $m R N A$. Exon 1 was found to contain sequences corresponding to the 5 ' end of the coding sequence in the full-length cDNA (Fig. 5). Exon 1 also contained nucleotide sequences upstream from the translation initiation codon, ATG, potentially corresponding to the $5^{\prime}$ untranslated segment of the mRNA. Computer analysis of the exon 1 sequences upstream from ATG codon identified a putative cap site consensus sequence, ACTTTT, at - [94-89] (27) (Fig. $3 A$ ). To map the 5' end of the mRNA, a PCR-based strategy was first devised to approximate the position of the $5^{\prime}$ end of the mRNA. Specifi- cally, four separate $5^{\prime}$ primers $(a-d)$ corresponding to the sequences surrounding the putative cap site were synthesized for PCR (Fig. $3 A$ ). These primers, together with a 3' primer (oligomer 2 in Fig. $3 A$ ) corresponding to nucleotide sequences in exon 3 of the BPAG1 mRNA, were used in RT-PCR. The position of the oligomer used for primer extension to generate a cDNA template for PCR from normal human keratinocyte RNA is shown in Fig. $3 A$ (oligomer 1 ). Analysis of the PCR products indicated that bands of expected size, 352, 372, and $402 \mathrm{bp}$, were detected with 5' primers a, b, and c, respectively, when cDNA derived from human keratinocyte mRNA was used as template (Fig. $3 \mathrm{~B}$ ). However, no band was detected with the 5' primer d in parallel PCR on the same cDNA. Bands of the expected size, varying from 750 to $820 \mathrm{bp}$, were detected with all four 5' primers when genomic DNA was used as template (Fig. $3 C$ ). Thus, the 5 ' end of the mRNA in keratinocytes appeared to be slightly upstream from the sequence that by computer analysis was predicted to be the cap site.

The precise position of the $5^{\prime}$ end of the mRNA was subsequently determined by primer extension analysis. Specifically, a reverse primer corresponding to the position -1 to -24 in BPAG1 cDNA was used for primer extension, as described in Methods. The product of primer extension was found to be $\sim 100$ bp in size (Fig. 4). This observation agrees with the PCR-based estimation of the position of the cap site. Thus, the sequence at - [94-89], as predicted by the computer analysis, is not the actual $5^{\prime}$ end of the mRNA, while the end is more likely
A

\section{$-144$ \\ $-94$}
ATGTTGAAGCATATTAAGGAGCCACTAAAAGCTGTAATATTCAGCTGCC

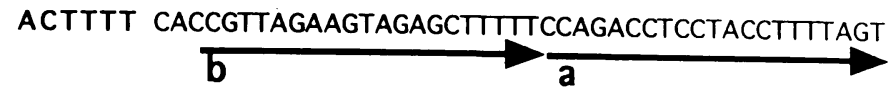
$-44$
CTACTTTGAAAGGTGAAAGAAAGAACATCGTTTCAGGAATAAAAATG

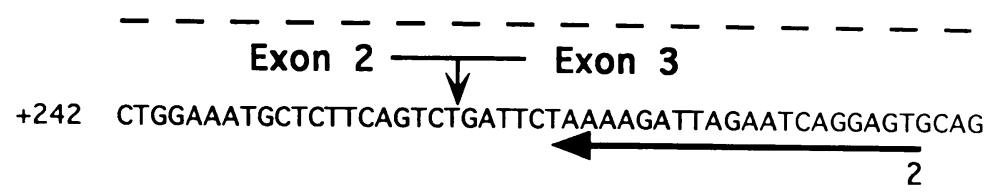
$+292$

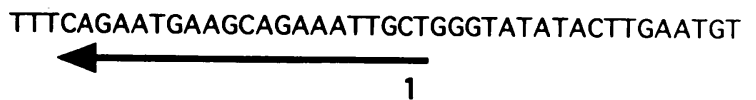
B

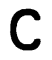
Figure 3. Strategy for identification of the $5^{\prime}$ end of the human BPAG1 mRNA. ( $A$ ) PCR primers surrounding the putative cap site (ACTTTT) at position - [94-89], as predicted by computer analysis, were synthesized (oligo- mers a-d). These 5' primers, together with a 3' primer (oligomer 2) corresponding to the nu- cleotide sequences in the exon 3 of the gene, were used for amplification of the correspond- ing regions by RT-PCR. The position of the primer (oligomer 1) used for generation of a cDNA from human keratinocyte RNA is indi- cated. $(B)$ Analysis of the PCR products by $1 \%$ agarose gel revealed that the reactions primed with 5 ' primers $a, b$, and c resulted in a product of the expected size of 352,372 , and $402 \mathrm{bp}$, respectively. No product was seen when the oligomer $d$ was used in PCR reaction. (C) Use of genomic DNA as template for the PCR reaction resulted in a product of the ex- pected size, $\sim 750$ to $820 \mathrm{bp}$, with all four pairs of primers. Lane $M$ contains molecular weight markers; lane $C$ is a control lane am- plified without template. 
to be in the position -101 . This conclusion was supported by previous studies that have shown that, with the exception of the histone genes, most eukaryotic genes contain their cap site 30 bp downstream from the first or second $\mathrm{T}$ in the TATA box, and the most likely $5^{\prime}$ nucleotide of the cap site is $A(27)$. Thus, the ultimate $5^{\prime} \mathrm{A}$ nucleotide of the cap site in the BPAG1 mRNA appears to reside at the position -101, which is exactly $30 \mathrm{bp}$ downstream from the second $\mathrm{T}$ in the putative TATA box (see Fig. $3 A$ ).

Exon 22 was shown to contain sequences corresponding to the carboxyl-terminal end of the deduced protein, as well as 928 nucleotides of the $3^{\prime}$ untranslated sequences, including a consensus motif for polyadenylation, AATAAA (28) (Fig. 2). Thus, the composite human BPAG1 mRNA, including the coding region and $5^{\prime}$ and $3^{\prime}$ untranslated segments, is calculated to be at least $8,977 \mathrm{bp}$ in size, excluding the poly-A tail. This size is consistent with the size of the corresponding mRNA, $\sim 9 \mathrm{~kb}$, as detected by Northern analysis (9). At this point, there is no evidence for additional polyadenylation signals in the downstream sequences or any evidence for size heterogeneity of the mRNA transcripts (9).

Characterization of the 5'-flanking DNA sequences. Sequencing of one of the genomic clones $(\lambda 1)$ for BPAGl identified the initiation codon (ATG) for translation (Fig. 5). Examination of the upstream flanking DNA up to $-2,637$ revealed the presence of features suggestive of a promoter. In particular, a variant of the consensus TATA(A/T)A sequence, TATTTA, was located in the position - [128-133], and a canonical CCAAT motif was identified in the position [-279283] (Fig. 5). The 38-bp segment immediately preceding the ATG codon has been previously identified in a BPAG1 cDNA, thus representing $5^{\prime}$ untranslated region of the corresponding mRNA (11). This 5' untranslated sequence contains stop codons for translation (either TGA or TAG) in all three reading frames, thus suggesting that the ATG in the position $+[1-3]$

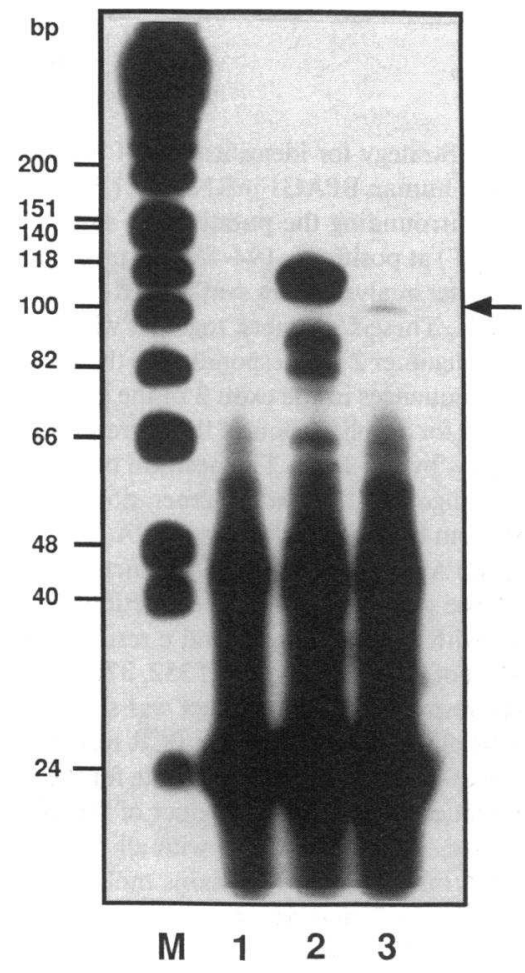

Figure 4. Primer extension analysis of the BPAG1 mRNA. Keratinocyte RNA ( $30 \mu \mathrm{g})$ was used as template for primer extension with an oligomer PE1 (see text). A band of $\sim 100$ bp is noted on lane 3 , which contained the complete reaction mixture. Lanes 1 and 2 show a negative control (RNA omitted) and a positive control (a 110 bp extension product from mRNA provided in the commercial kit), respectively. Lane $M$ contains molecular weight markers of the $\phi X$ 174Hinf I digests. codes for the first amino acid (Met) of the translated protein. These features suggested that the region extending from -1 to $-2,637$ contains the promoter for BPAG1 gene.

Further elucidation of the upstream sequences revealed the presence of several cis-regulatory consensus motifs. Specifically, there were three AP-1 (29) and three AP-2 (30) putative binding sites within the region extending from $-1,041$ to $-2,303$ (Fig. 5). Furthermore, the presence of a retinoic acid responsive element (RARE) (31), a cAMP responsive element (CRE) (32), and eight glucocorticoid responsive elements (GRE) $(33,34)$ were noted (Fig. 5). The functionality of these consensus motifs remains to be tested.

Expression of BPAG1 promoter/CAT reporter gene construct in cultured cells. To test for the functional promoter activity of the 5'-flanking region of the BPAGl gene, several chimeric promoter/CAT reporter gene plasmids were constructed (see Methods). The largest of them, pBP2.6CAT, extended from -1 to $-2,637$. Six additional 5 ' deletion constructs were developed, as described in Methods and in Fig. 6. These constructs were then utilized in transient transfections, first in cultures of human epidermal keratinocytes that have been previously shown to express the BPAG1 gene, as detected at the mRNA level $(9,35)$. Assay of CAT activity in cells transfected with these constructs revealed clearly detectable activity, while essentially no activity was detected in cells transfected in parallel with pBSOCAT as a negative control. The activity observed in parallel transfections of keratinocytes with the seven different constructs revealed reproducible variability, after correction of the CAT activities by the $\beta$-galactosidase activity in the same samples (Fig. 6). However, the variances were relatively small, with the exception of pBP0.9CAT, which consistently showed about five- to eightfold higher activities than those noted with pBP0.7CAT and pBP1.4CAT constructs (Fig. 6). These observations suggested the presence of upregulatory, enhancer-like elements within the regions - [717-924], and the possible presence of downregulatory, silencer-like elements within the regions $-[924-1,427]$; however, these regions were not examined in further detail.

Evidence for tissue specific expression. The 230-kD BP antigen is expressed exclusively in the cells of stratifying squamous epithelia, including epidermal keratinocytes (2). In this study, we first examined the expression of the BPAG1 gene at the mRNA level by Northern hybridizations with RNA isolated from a variety of normal and transformed cells of epithelial or mesenchymal origin. As indicated in Fig. 7, a clear signal corresponding to the $9 \mathrm{~kb}$ mRNA transcript of BPAGl was detected in normal human keratinocytes (NHK), while no expression could be noted in NIH3T3, HeLa, WISH, or KB cell cultures. Extended exposure ( up to $5 \mathrm{~d}$ ) of the filter to the $\mathrm{x}$-ray film did not reveal the presence of a mRNA transcript of the same size in the latter cultures (not shown). Rehybridization of the same Northern filter with the GAPDH cDNA revealed a clearly detectable, relatively even, signal of $\sim 1.3 \mathrm{~kb}$ in all lanes (Fig. 7). Thus, the BPAG1 gene is expressed exclusively in normal epidermal keratinocyte cultures maintained in low $(0.15 \mathrm{mM}) \mathrm{Ca}^{2+}$-containing serum-free medium, conditions that allow the maintenance of the basal keratinocyte phenotype.

Recently, two nucleotide sequences potentially conferring tissue-specific expression of several genes in epidermal cells have been identified. One of these sequences, 5'-AARCCAAA$3^{\prime}$, designated as a CK-8mer, has been identified in a number of 
-2637 TCTAGAACAC ACTCAGTAAA TGTTAGTTCA GGTTCCCTTT CTGCCACGCT TCTACAAGTG

- 2577 GATTTACTCC ATGAAATCAT CACTAGTTCT CTGTCATTTT GTCTCTCACT ACATCCAGTT -2517 GCCCAGGAAG GTACCATTTA GTCTTCAGGA AATGCTTGTT GGATGAATTT GTCTCCCTTA

-2457 CAGTCTTAAC CTGGACCTGG GAATTGTGAT CTGTGTTCCT CATTAGCCAT GAAGCTCATA - 2397 GGACCTAGAC CTTGCAGGGT GTATTAGTCC ATTCTCACGC TGCTAATAAA GACATATCTG -2337 AGACTGGGTA ATTTATAAAG GAAAGAGTT TAATTGACTC ACAGTTACAT ATGACTGGAG -2277 AGGCCTCACA ATCATCCTCC AAGGCGAATG AGGAGCAAAG TCACATCTTA CATGTCAGCA

-2217 GGCAAGAGCT TGTGCAGGGG AACTCCATTT ACAAAACCGT CAGATCTCTT GAGACTTATT -2157 CATTACCATG AGAACAGTAT GGGGGAAATC GCCCCCGTGA TTCAGTTATC TCCACCTGGC -2097 CCCACAGCTT GACACATGAG GATTATTACA ATTCAAGATG CGATTTGGGT GGGGACACAG - 2037 GCAAACCATA TTTACAGGGA ATGGCCTGTG TGCCAGACAC ACCTCTCCAG CCCCCTTGCT

- 1977 CTCTGCACAG TAGCCACACT GGTGCCTTAC ACCTAAACAT TCTCAGATCT CAGCTCCAAC - 1917 ATTAGTTTTC TCGAGAAACT TTCCATGGI CCCTAAGGCT AGTTCAGATA TGTTGCTTAG

-1857 AACTCTCATG TTTCCATGTA TTGTTCCTCT ATAGTGCTTA TGTTGGTTGT ATTTTTATAT

- 1797 TTCGGTGATT GCTTAAGTCT TGCCTATTCC ACAGATATGA GCTCCAACAA TGTGGAGAGA

- 1737 AACCCTTCTT TTTTTTCTGT CCCTTGCGTT GCCAGTGCTT TACACAGCGC CTGACACAAA

- 1677 CATTAGAGGT GCTCAGTATT GTCATGTTCT CTGTGCCTTA CACATÍGACT TCA]GGATTCA

- 1617 ACGTTGTGAA ATAATGCATA AAATAAATAT ATGAGAGCAT GTCTAACCTG TCAGTCCTTT

- 1557 ATTAAATTAC TGATTACATT TATGTTAAAG TATAATAATA TGTGTACATA ACTGGATTAT

- 1497 TA ACCAAA ACAAGCCATA AATATACAGG ATGCAGCTTT TACGGAAAG GTAATTTGAG

- 1437 TGATTGAAAT GAGATACTAT GTTAATCATT TGAAATAATG GAAAAGTATT CCAGAGGATA

- 1377 CAGAGTAGAT TTTTAGTCAT CAGAACAGTA GCTGAATTTT AAGATATGTT CATAAAAGAA

- 1317 AGCAGAGAGT GGTTGAGAGT TCAGGGCCTA GAAGCCACTC AATTTTAAAT TAGATTATTA

- 1257 GCTCAGCCTG CAAAGTTGTA TGATGTTAGA CAAGTTCTTT AACCGTCTAA GCCTCAGTTT

- 1197 CTTCATTTGT AAACAGGATG TAATAGTGCC TACTACATTG GGTGGTTATG AGTATGAGAT

- 1137 GAGTTATGTA TCTAAAATGA TTAATTTCTT GCACATAATA ATTACTTAAT AAATGCCAGG

- 1077 TGCCACTGTT ATTGTTTTGA GCTATTCTGT TCACTCACTA TATTTGATGT GGCACTCTCA

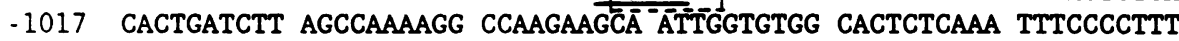

- 957 CTTGACCCCT GAAAAGAAAA ATATCTATTC TTAGAGTGGA GAAGTAACAG TATTATTGGA

- 897 GGTAGGAAAA TATTTAGGAG GCTGGTTGAT AAATGCCTTC ATGTTTCTTG ATGCGAATTT

- 837 TTTGGCATTG TCTATGTATT GCGAGTAATA GCTATGTGGC ATCCAAGTGT ATCAAAAACA

- 777 AATGGGAAAG AGCCAGTGTT CTTATGCCAT GTAATATCCT ATAGAATGGA AAAACAAGCA

- 717 CAGACAAATT AAATTTGTAA GCATTGCATC AGTATTCTAC TTGGCACTTT ATAGTAGGTT

- 657 TAGAAGTAAC ATTACTGGGA GCGTGTCATA TAAAGAAAGg TAAATGGATA CTTTTTCCAT

- 597 TAATTTAACC TAATAAACAA ATGACATGGT GGTTAAAATG GAAGCGTACT TTGCTTCTCA

- 537 TCAAATTCAT TTGTTTATAG GTACTTCATA ATTTCTAGCT GTTTGCCTTC CTTACATTAT

- 477 TCAGCACAGA CCTGGTCTTT TGCAGAAGTC AGACTATGAT TGGATATTTG TGTTCTTTTA

- 417 GCTAATGATT GGTATTGCCA GAATAATCTT TACTAGTTGT CCTGGACATC TGTGAGGCAT

- 357 CTGAGTTGTT TTTTAGGTGG TTGCAATTGG ATGACTCTTA GTGCAAATAT TTGGCATATC

- 297 CTTTCCAGTG ACGCČČÅATC TGCCTGCTTT GTTCATACTT GTAGGGTTTT AATACAAGCT

- 237 ACAAAAGGCA AACCTCAGCT AGCAAACGAA TGGCTTTAGG GTAGATTCTT TTGAATAGTT

- 177 ATCAGCATAT TATTTGCTGT GTGTTGCATA TGCATGTTGA AGCATATTTA AGGAGCCACT

- 117 AAAAGCTGTA ATATTCAGCT GCCACTTTTC ACCGTTAGAA GTAGAGCTTT TTCCAGACCT

57 CCTACCTTTT AGTCTACTTT GAAAGGTGAA AGAAAGAACA TCGTTTCAGG AATAAAA

+1 ATG CAC AGT AGT AGT TAT AGT TAC CGT AGC AGT GAT TCT GTG TTT MET His Ser Ser Ser Tyr Ser Tyr Arg Ser Ser Asp Ser Val Phe

+46 AGT AAC ACT ACC AGC ACT CGA ACC AGT CTT GAT TCA AAT GAA AAT Ser Asn Thr Thr Ser Thr Arg Thr Ser Leu Asp Ser Asn Glu Asn

+91 CTT CTC TTG GTT CAT TGT GGT CCA ACA CTG ATC AAC TCT TGC ATT Leu Leu Leu Val His Cys Gly Pro Thr Leu Ile Asn Ser Cys Ile

+136 AGC TTC GGC AGT GAA TCC TTT GAT GGA CAC AG gtattgaatcattgg Ser Phe Gly Ser Glu Ser Phe Asp Gly His
Figure 5. Nucleotide sequences corresponding to the $5^{\prime}$ end of the human BPAG1 gene and the deduced amino acid sequence of exon 1 . Note the presence of a methionyl residue corresponding to the nucleotide position +1 to +3 , followed by a 55-amino acid peptide encoded by exon 1. The $3^{\prime}$ end of the exon 1 contains two nucleotides correspond-ing to a split codon for $\operatorname{Arg}(\underline{A G G})$. Note the presence of a putative TATAAA box (double underline) and CCAAT box (dots above). Several additional putative cis-regulatory elements were identified in the $5^{\prime}$-flanking region, including three AP-1 $(\square)$, three AP-2 $(\sqcup)$ sites, a retinoic acid responsive element ( $(i-)$, a cAMP responsive element ([ ]), and eight glucocorticoid responsive elements (underlined). Also, note the presence of two segments that may confer keratinocyte-specific expression to the gene, CK-8mer ( $\square$ ) and the KTF-1 binding site $(\square)$. The putative transcription initiation site is indicated $(\boldsymbol{\nabla})$. The DNA sequences reported here have been deposited in the GenBank data base under accession no. L08972. keratin genes, as well as in the gene for human involucrin (3638 ). An identical segment in the 5'-flanking region of the human BPAGl gene was detected in the position - $[1,488-$ $1,495]$; we designated this motif as Keratinocyte Responsive Element-1 (KRE1). A second sequence, 5'-ACCCTGAGGCT-3', previously described as the binding site for Keratinocyte Transcription Factor-1 (KTF1), has been identified in xenopus epidermal keratin gene (39). The corresponding trans-acting factor was subsequently shown to be identical or closely related to AP2 (40-42). Examination of the 5'-flanking sequences in human BPAGl gene identified a nucleotide se- quence highly homologous, but not identical, to the KTF1 binding site in the position - $[1,878-1,888]$. This sequence in the BPAG1 promoter was 5'-TCCCTAAGGCT-3', and was designated as KRE2. These observations suggested that the construct pBP1.9CAT may contain elements conferring tissuespecific expression of the BPAG1 gene in keratinocytes.

To examine the tissue specific expression of the BPAG1 gene at the transcriptional level, several cell types, one being expressing (normal human epidermal keratinocytes) and the other ones being nonexpressing (KB, WISH, HeLa and NIH $3 \mathrm{~T} 3$ cells; see Fig. 7), as well as normal human skin fibroblasts 


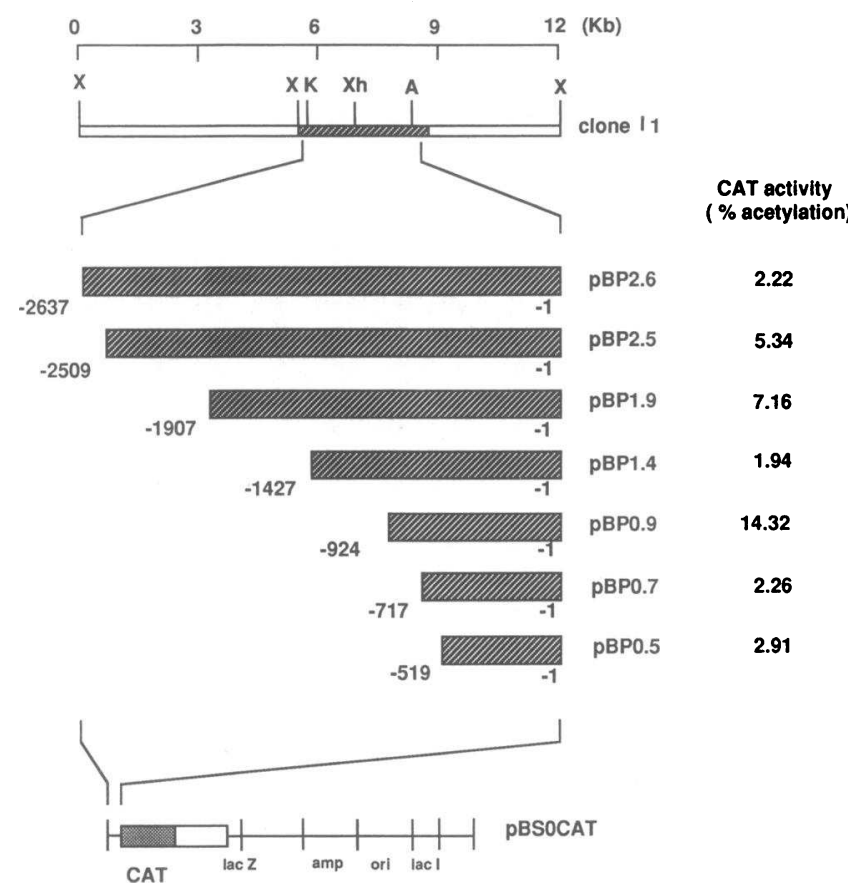

Figure 6. Construction of a 5' deletion library of the BAPG1 5'flanking region linked to the CAT promoter gene and assay of CAT activity in human epidermal keratinocytes transfected with BPAG1 promoter/CAT constructs. The construction of individual DNA fragments corresponding to the 5'-flanking region of the BPAG 1 gene, is described in Methods. These DNA fragments were cloned into a multiple cloning site in pBSOCAT, a promoterless construct containing the entire CAT gene, small $t$ intron, and polyadenylation signal in plasmid Bluescript. Seven different $5^{\prime}$ deletion constructs containing the promoter region with their $3^{\prime}$ end at -1 and the $5^{\prime}$ end varying from $-2,637$ to -519 were transfected in parallel. The CAT activity was determined by separation of acetylated and nonacetylated $\left[{ }^{14} \mathrm{C}\right]-$ chloramphenicol derivatives by thin-layer chromatography. Each construct was examined in two parallel cultures. The CAT activity, expressed as percent acetylation, is indicated on the right (mean of the two parallel transfections of each construct after correction for transfection efficiency by $\beta$-galactosidase activity in the same samples). The values represent the activity above the control value noted in cells transfected with the promoterless pBSOCAT.

that were previously shown not to express this gene (9), were transfected in parallel with the pBP1.9CAT. As indicated above, this construct contains the two putative sequences potentially conferring tissue specificity of expression. The results indicated a high level of expression in normal human keratinocytes, while the promoter activity in other cells tested in parallel was less than $10 \%$ of that noted in keratinocytes, after correction for $\beta$-galactosidase activity as an index of the transfection efficiency (Fig. 8). Thus, $\sim 1.9 \mathrm{~kb}$ of 5 '-flanking sequences of the BPAG1 gene appears to contain cis-elements which confer tissue specificity to the expression of the gene. This hypothesis was further tested by transient transfections of normal human keratinocytes, HeLa cells and NIH-3T3 fibroblasts in parallel with the pBP1.9CAT and pBP0.5CAT constructs. The latter construct is devoid of the putative cis-elements that may confer the tissue specific expression to the BPAG1 gene. The results indicated that the promoter activity with the $\mathrm{pBP} 1.9 \mathrm{CAT}$ construct was significantly higher than the activity noted with pBP0.5CAT when transfected into normal human keratinocytes ( Table I). In contrast, similar levels of activities with both
Figure 7. Demonstration of the BPAGl gene expression in normal human keratinocytes by Northern analysis. Total RNA was isolated from normal human keratinocytes (NHK), mouse NIH-3T3, HeLa, WISH or KB cells in culture. The Northern filter was first hybridized with a human 1.7 kb BPAG1 cDNA (top). Note a clear signal with the NHK RNA corresponding to the 9 kb BPAG1 mRNA. The same filter was subsequently hybridized with a GAPDH cDNA (bottom) indicating the presence of this ubiquitously expressed $1.3 \mathrm{~kb}$ mRNA in all lanes in approximately even amount. Thus, normal human epidermal keratinocytes selectively express the BPAG1 mRNA.

constructs were noted in HeLa cells, while the activity of the pBP1.9CAT was about one third of that noted with pBP0.5CAT when NIH-3T3 cells were used for transfection (Table I). The role of one of the putative tissue specific elements, KRE2, which has been previously suggested to confer tissue specificity to the expression of the human keratin 14 gene $(41,42)$, was examined in further detail. Specifically, an oligonucleotide fragment consisting of 5'-ATGGTTCCCTAAGGCTAGTTC-3' (positions -1893 to -1873 in Fig. 5) and

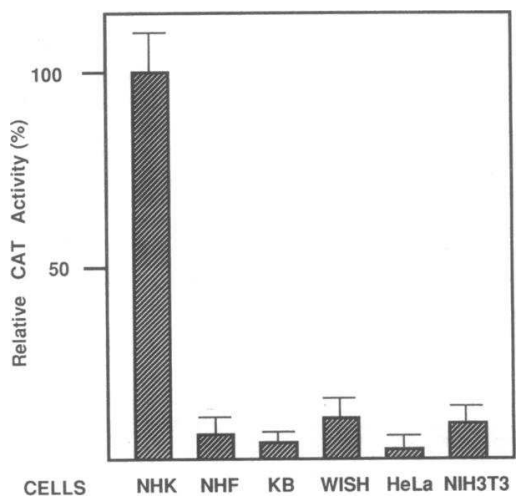

Figure 8. Relative promoter activity of the pBP1.9CAT construct in transient transfection of cultured cells. Cultured cells were cotransfected with the pBP1.9CAT construct containing $1.9 \mathrm{~kb}$ of the 5 '-flanking DNA with putative tissue specific cis-elements and with SV $2 \beta$ Gal construct. Equal aliquots of the cell extracts were assayed for CAT activity. The values are expressed as the activity of CAT after correction for $\beta$-galactosidase activity, and the relative activity in NHK was designated as $100 \%$. Note that the BPAG1 promoter activity in NHF, human epidermal carcinoma cells (KB), human transformed amniotic epithelial cells (WISH), HeLa, or mouse transformed fibroblasts (NIH-3T3) was $<10 \%$ of that noted in NHK cultures. The values are expressed as mean $+\mathrm{SD}$ of triplicate cultures transfected in parallel. 
Table I. Relative Promoter Activities of the pBP1.9CAT and pBP0.5CAT Constructs in Transient Transfections of NHK, HeLa or NIH-3T3 Cells in Culture

\begin{tabular}{|c|c|c|c|c|}
\hline & \multirow[b]{2}{*}{ Construct } & \multicolumn{3}{|c|}{ CAT activity in cell cultures* } \\
\hline & & NHK & HeLa & NIH-3T3 \\
\hline Promoter activity & pBP1.9CAT & 7.16 & 0.91 & 0.07 \\
\hline (\% Acetylated) & pBP0.5CAT & 2.75 & 1.41 & 0.23 \\
\hline $\begin{array}{l}\text { Relative activity } \\
\text { (1.9CAT/0.5CAT) }\end{array}$ & & 2.60 & 0.64 & 0.29 \\
\hline
\end{tabular}

* NHK, HeLa and NIH-3T3 cell cultures were transfected in parallel with the BPAG1 promoter/CAT constructs indicated, together with SV $2 \beta G a l$ construct, as described in the text and in Figure 8. The values are mean of two parallel determinations after correction for the $\beta$-galactosidase activity in the same cell extracts.

\$ The values represent ratios of the activities obtained with pBP1.9CAT and pBP0.5CAT constructs, as determined from the mean values noted for each cell type.

containing the KRE2 binding sequence (underlined), was ligated in front of the pBP1.4CAT construct to generate the construct pKRE2/BP1.4CAT (Fig. 9). Parallel transfections with pBP1.4CAT and pKRE2/BP1.4CAT constructs showed that there was no difference in the CAT activity when human skin fibroblast cultures were used in transient transfections (Fig. 9). In contrast, the construct containing the KRE2 element showed about threefold higher activity in human epidermal keratinocytes, in comparison to the unmodified pBP1.4CAT construct. The activity noted with the pKRE2/BP1.4CAT was

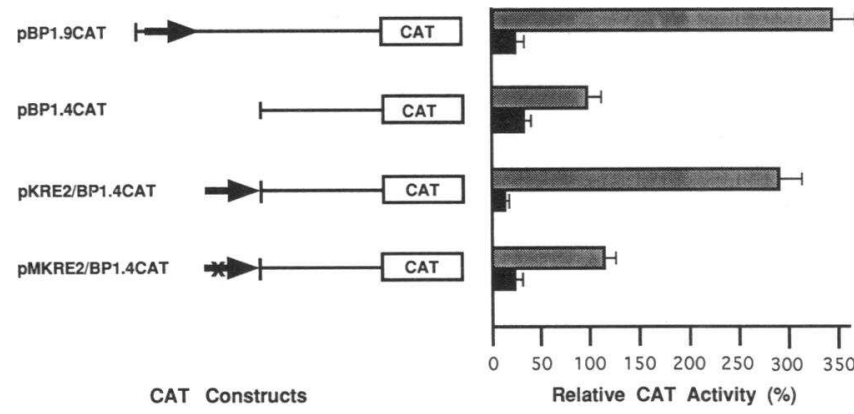

Figure 9. Demonstration that the KRE2 element provides tissue specificity for the BPAG1 gene expression. The construct pBP1.9CAT containing the KRE2 sequence $(\rightarrow)$ at the position $-[1,878$ to 1,888 ], and pBP1.4CAT were transfected into NHK $(\square)$ or NHF $(\square)$ in culture. Note the $\sim 3.5$-fold higher activity with the pBP1.9CAT construct in keratinocytes while no significant difference in fibroblasts was noted, after correction of the CAT activity to the $\beta$-galactosidase activity in the same cell extracts. Cloning of a 21-bp sequence containing the KRE2 element $(\rightarrow)$ in front of BP1.4CAT also resulted in 3.0-fold higher activity in keratinocytes, but not in fibroblasts, in comparison to pBP1.4CAT. A mutated construct, pMKRE2/ BP1.4CAT, in which two $\mathrm{C} \rightarrow \mathrm{A}$ substitutions were made in the KRE2 sequence (see text), showed activity similar to that of pBP1.4CAT. The values are expressed as relative CAT activity, as a percent of the activity noted with pBP1.4CAT in NHK, and the values are mean \pm SD from 2 or 3 parallel samples in 3-4 separate experiments, after correction of the activity for $\beta$-galactosidase activity in the same cell extracts. approximately at the same level as was obtained with the pBP1.9CAT construct in parallel transfections (Fig. 9). To examine the role of KRE2 in further detail, a similar construct that contained a mutated KRE2 sequence, 5'-ATGGTTCAATAAGGCTAGTTC-3' (the $C$ to A substitutions underlined), was generated. This construct, pMKRE2/BP1.4CAT, did not demonstrate elevated activity, as compared with pBP1.4CAT (Fig. 9). Thus, these observations provide direct evidence for the role of the KRE2 sequence in tissue specific expression of the BPAG1 gene.

In conclusion, we have elucidated the exon-intron organization of the entire human BPAGl gene coding for a $230-\mathrm{kD}$ protein, the major autoantigen in $\mathrm{BP}$, an acquired autoimmune disease. Furthermore, we have characterized regulatory elements in the promoter region of the gene, and identified $5^{\prime}$ segments potentially providing tissue specificity of the expression. The development of these promoter/reporter gene constructs now provides a means to study the transcriptional regulation of BPAGl gene by cytokines and growth factors, such as transforming growth factor- $\beta$, which has been shown to modulate the corresponding gene expression at the mRNA level (35). Understanding of the structure and regulation of the BPAG1 should facilitate not only elucidation of BP, but also the search and identification of mutations in heritable diseases affecting the cutaneous basement membrane zone, such as epidermolysis bullosa (7).

\section{Acknowledgments}

We thank Debra Pawlicki for expert secretarial help, and Huiping Qin and Lin Lin for technical assistance. Dr. James W. Fox, IV, and Dr. John H. Moore, Jr., provided tissue for cell cultures.

This work was supported in part by U.S. Public Health Service, National Institutes of Health grants 5-PO1-AR38923 and T32AR7561, and the Dermatology Foundation.

\section{References}

1. Lever, W. F. 1965. Pemphigus and Bullous Pemphigoid. Charles C. Thomas, Springfield, IL. 75-103.

2. Stanley, J. R., P. Hawley-Nelson, S. H. Yuspa, E. M. Shevach, and S. I. Katz. 1981. Characterization of bullous pemphigoid antigen: a unique basement membrane protein of stratified squamous epithelia. Cell. 24:897-903.

3. Mueller, S., V. Klaus-Kovtun, and J. Stanley. 1989. A 230-kD basic protein is the major bullous pemphigoid antigen. J. Invest. Dermatol. 92:33-38.

4. Morrison, L. H., R. S. Labib, J. J. Zone, L. A. Diaz, and G. J. Anhalt. 1988. Herpes gestationis autoantibodies recognize a $180 \mathrm{kD}$ human epidermal antigen. J. Clin. Invest. 81:2023-2026.

5. Westgate, G. E., A. C. Weaver, and J. R. Couchman. 1985. Bullous pemphigoid antigen localization suggests an intracellular association with hemidesmosomes. J. Invest. Dermatol. 84:218-224.

6. Mutasim, D. F., Y. Takahashi, R. S. Labib, G. J. Anhalt, H. P. Patel, and L. A. Diaz. 1985. A pool of bullous pemphigoid antigen is intracellular and associated with the basal cell cytoskeleton-hemidesmosome complex. J. Invest. Dermatol. 84:47-53.

7. Uitto, J., E. A. Bauer, and A. N. Moshell. 1992. Symposium on epidermolysis bullosa: molecular biology and pathology of the cutaneous basement membrane zone. J. Invest. Dermatol. 98:391-398.

8. Stanley, J. R., T. Tanaka, S. Mueller, V. Klaus-Kovtun, and D. Roop. 1988. Isolation of complementary DNA for bullous pemphigoid antigen by use of patients' autoantibodies. J. Clin. Invest. 82:1864-1870.

9. Sawamura, D., K. Li, K. Nomura, Y. Sugita, A. M. Christiano, and J. Uitto. 1991. Bullous pemphigoid antigen: cDNA cloning, cellular expression, and evidence for polymorphism of the human gene. J. Invest. Dermatol. 96:908-915.

10. Tanaka, T., D. D. Parry, V. Klaus-Kovtun, P. M. Steinert, and J. R. Stanley. 1991. Comparison of molecularly cloned bullous pemphigoid antigen to desmoplakin I confirms that they define a new family of cell adhesion junction plaque proteins. J. Biol. Chem. 266:12555-12559.

11. Sawamura, D., K. Li, M.-L. Chu, and J. Uitto. 1991. Human bullous pemphigoid antigen (BPAG1): amino acid sequences deduced from cloned 
cDNA predict biologically important peptide segments and protein domains. $J$. Biol. Chem. 266:17784-17790.

12. Sawamura, D., K. Nomura, Y. Sugita, M.-G. Mattei, M.-L. Chu, R. Knowlton, and J. Uitto. 1990. Bullous pemphigoid antigen: cDNA cloning and mapping of the gene to the short arm of human chromosome 6. Genomics 8:722726.

13. Green, K. J., D. A. D. Parry, P. M. Steinert, M. L. A. Virata, R. M. Wagner, B. D. Angst, and L. A. Nilles. 1990. Structure of the human desmoplakins: implication for function in the desmosomal plaque. J. Biol. Chem. 265:2603-2612.

14. Sambrook, J., E. F. Fritsch, and T. Maniatis. 1989. Molecular Cloning: A Laboratory Manual. 2nd ed. Cold Spring Harbor Laboratory, Cold Spring Harbor, NY. 9.31-9.57.

15. Kerr, C., and P. D. Sadowski. 1972. Gene 6 exonuclease of bacteriophage T7; purification and properties of the enzyme. J. Biol. Chem. 247:311-318.

16. Sanger, F., S. Nicklen, and A. R. Coulson. 1977. DNA sequencing with chain-terminating inhibitors. Proc. Natl. Acad. Sci. USA. 74:5463-5467.

17. Fazio, M. J., V.-M. Kähäri, M. M. Bashir, B. Saitta, J. Rosenbloom, and J. Uitto. 1990. Regulation of elastin gene expression: evidence for functional promoter activity in the 5'-flanking region of the human gene. J. Invest. Dermatol. 94:191-196.

18. Boyce, S. T., and R. G. Ham. 1983. Calcium-regulated differentiation of normal human epidermal keratinocytes in chemically defined clonal culture and serum-free serial culture. J. Invest. Dermatol. 81:33S-40S.

19. Olsen, D. R., N. J. Hickok, and J. Uitto. 1990. Suppression of ornithine decarboxylase gene expression by retinoids in cultured human keratinocytes. $J$. Invest. Dermatol. 94:33-36.

20. Behr, J.-P., B. Demeneix, J.-P. Loeffler, and J. Perez-Mutul. 1989. Effcient gene transfer into mammalian primary endocrine cells with lipopolyaminecoated DNA. Proc. Natl. Acad. Sci. USA. 86:6982-6986.

21. Gorman, C. M., L. F. Moffat, and B. H. Howard. 1982. Recombinant genomes which express chloramphenicol acetyl transferase in mammalian cells. Mol. Cell. Biol. 2:1044-1051.

22. Chomczynski, P., and N. Sacchi. 1987. Single-step method of RNA isolation by acid guanidinium thiocyanate-phenol-chloroform extraction. Anal. Bio chem. 162:156-159.

23. Padgett, R. A., P. J. Grabowski, M. M. Konarska, S. Seiler, and P. A. Sharp. 1986. Splicing of messenger RNA precursors. Annu. Rev. Biochem. 55:1119-1150.

24. Ullu, E., and C. Tschudi. 1984. Alu sequences are processed 7SL RNA genes. Nature (Lond.). 312:171-172.

25. Jelinek, W. R., and C. W. Schmid. 1982. Repetitive sequences in eukaryotic DNA and their expression. Annu. Rev. Biochem. 51:813-844.

26. Ryynänen, M., R. G. Knowlton, D. Sawamura, K. Li, G. Giudice, L. A. Diaz, and J. Uitto. 1991. Bullous pemphigoid antigens (BPAGs): identification of RFLPs in human BPAG1 and BPAG2, and exclusion as candidate genes in a large kindred with dominant epidermolysis bullosa simplex. Genomics. 11:10251029.
27. Sadler, J. R., M. S. Waterman, and T. F. Smith. 1983. Regulatory pattern identification in nucleic acid sequences. Nucleic Acids Res. 11:2221-2231.

28. Birnstiel, M. L., M. Busslinger, and K. Strub. 1985. Transcription termination and 3' processing: the end is in site. Cell. 41:349-359.

29. Angel, P., M. Imagawa, R. Chiu, B. Stein, R. J. Imbra, H. J. Rahmsdorff, C. Jonat, P. Herrlich, and M. Karin. 1987. Phorbol ester-induced genes contain a common cis-element recognized by a TPA-modulated trans-acting factor. Cell. 49:729-739.

30. Williams, T., and R. Tjian. 1991. Analysis of the DNA-binding and activation properties of the human transcription factor AP-2. Genes \& Dev. 5:670682.

31. de Thé, H., M. del Mar Vivanco-Ruiz, P. Tiollais, H. Stunnenberg, and A. Dejean. 1990. Identification of a retinoic acid responsive element in the retinoic acid receptor $\beta$ gene. Nature (Lond.). 343:177-180.

32. Roesler, W. J., G. R. Vandenbark, and R. W. Hanson. 1988. Cyclic AMP and the induction of eukaryotic gene transcription. J. Biol. Chem. 263:90639066.

33. Evans, R. M. 1988. The steroid and thyroid hormone receptor superfamily. Science (Wash. D.C.). 240:889-895.

34. Beato, M. 1989. Gene regulation by steroid hormones. Cell. 56:335-344.

35. Sollberg, S., J. Ryynänen, D. R. Olsen, and J. Uitto. 1992. Transforming growth factor- $\beta$ up-regulates the expression of the genes for $\beta 4$ integrin and bullous pemphigoid antigens (BPAG1 and BPAG2) in normal and transformed human keratinocytes. J. Invest. Dermatol. 99:409-414.

36. Blessing, M., H. Zentgraf, and J. L. Jorcano. 1987. Differentially expressed bovine cytokeratin genes. Analysis of gene linkage and evolutionary conservation of 5'-upstream sequences. EMBO (Eur. Mol. Biol. Organ.) J. 6:567575.

37. Blessing, M., J. L. Jorcano, and W. W. Franke. 1989. Enhancer elements directing cell-type-specific expression of cytokeratin genes and changes of the epithelial cytoskeleton by transfections of hybrid cytokeratin genes. $E M B O$ (Eur. Mol. Biol. Organ.) J. 8:117-126.

38. Cripe, T. P., T. H. Haugen, J. P. Turk, F. Tabatabai, P. G. Schmid II, M. Durst, L. Gissmann, A. Roman, and L. P. Turek. 1987. Transcriptional regulation of the human papilloma virus-16 E6-E7 promoter by a keratinocyte-dependent enhancer, and by viral $\mathrm{E} 2$ trans-activator and repressor gene products: implications for cervical carcinogenesis. EMBO (Eur. Mol. Biol. Organ.) J. 6:37453753.

39. Snape, A. M., E. A. Jonas, and T. D. Sargent. 1990. KTF-1, a transcriptional activator of Xenopus embryonic keratin expression. Development (Camb.) 109:157-165.

40. Snape, A. M., R. S. Winning, and T. D. Sargent. 1991. Transcription factor AP-2 is tissue-specific in Xenopus and is closely related or identical to Keratin Transcription Factor 1 (KTF-1). Development (Camb.) 113:283-293.

41. Leask, A., M. Rosenberg, R. Vassar, and E. Fuchs. 1990. Regulation of a human epidermal keratin gene: sequences and nuclear factor involved in keratinocyte-specific transcription. Genes \& Dev. 4:1985-1998.

42. Leask, A., C. Byrne, and E. Fuchs. 1991. Transcription factor AP-2 and its role in epidermal-specific gene expression. Proc. Natl. Acad. Sci. USA. 88:79487952. 